\title{
Sketch Based Image Retrieval Approach Using Gray Level Co-Occurrence Matrix
}

\author{
K."Nagarjuna Reddy \\ JNT University, \\ LIET, Himayatsagar, Hyderabad-8, \\ India
}

\author{
P. Prasanna Kumari \\ JNT University, \\ LIET, Himayatsagar, Hyderabad-8, \\ India
}

\begin{abstract}
This work focuses on Content Based Image Retrieval (CBIR) system using sketches, which is one of the most accepted, rising research areas of the digital image processing. The majority of the available image searching tools, such as Google Images and Yahoo Image search, are based on textual annotation of digital images. In these searching tools, images are manually annotated with keywords and then retrieved using text-based search techniques. The presentations of these systems are not satisfactory. The aim of CBIR is to extract visual content of an image automatically, like color, texture, or shape. The Proposed method used to introduce the design and the creation of CBIR systems, which is based on a free hand sketch which is known as Sketch based image retrieval (SBIR). In this technique, texture is used as feature for image retrieval. The texture features are obtained by using Gray-Level Co-occurrence Matrix (GLCOM).This process can be used as coarse level in hierarchical CBIR that reduces the database size from very large set to a small one. This small database can further be examined thoroughly using the wavelets, edge detection, etc. The sketch based system allows users an intuitive access to searching tools. This process can be implemented and simulated in MATLAB.
\end{abstract}

Keywords: Image, Gray level co-occurrence matrix (GLCOM).

\section{INTRODUCTION}

The spreading of information technology (IT) a huge data had to be managed, processed and stored. It was also textual and visual data. Parallelly of the look and quick evolution of computers an increasing measure of data had to be managed. The growing of data storages and revolution of internet had changed the world. The efficiency of searching in information set is a very important point of view. In case of texts keywords are searched flexibly, but if images the used, we cannot apply dynamic methods. Two questions can come up. The first is who yields the keywords. And the second is an image can be well represented by keywords. The human is able to recall visual information more easily using for example the shape of an object, or arrangement of colors and objects [2]. Since the human is visual type, images using other images are observed, and follow this approach also at the categorizing. In this case some features are searched for images, and these features are the keywords. At this moment unfortunately there are not frequently used retrieval systems, which retrieve images using the non-textual information of a sample image.

CBIR is a technique used for extracting similar images from an image database. This technology conquers the defects of traditional text-based image retrieval technology, such as heavy workload and strong subjectivity. It makes full use of image content features (color, texture, shape, etc.), which are analyzed and extracted automatically by computer to achieve the effective retrieval [1]. The purpose is to develop a content based image retrieval system, which can retrieve using sketches in frequently used databases. Using a sketch based system can be very important and efficient in many areas of the life. In some cases we can recall our minds with the help of figures or drawing [7].
The CBIR systems have a big significance in the criminal investigation. The identification of unsubstantial images, tattoos and graffities can be supported by these systems. Another possible application area of sketch based information retrieval is the searching of analog circuit graphs from a big database. The user has to make a sketch of the analog circuit, and the system can provide many similar circuits from the database $[5,6]$. In this work sketch based image retrieval system was developed by using GLCOM. The Texture Feature for retrieving the images by using GLCOM method are used [8].

\subsection{Texture Feature Extraction}

GLCOM creates a matrix with the directions and distances between pixels, and then extracts meaningful statistics from the matrix as texture features. The main benefit of GLCOM is its rotation invariance feature. It means the GLCOM propertity values of rotation form of an image are equal to its original image. GLCOM texture features normally used are shown in the following: GLCOM is composed of the probability value, it is defined by $P(\mathrm{i}, j \mid d, \theta)$ which expresses the probability of the couple pixels at $\theta$ direction and $d$ interval. When $\theta$ and $d$ is determined, $P(\mathrm{i}, j \mid d, \theta)$ is showed by $P i, j$. $P$ articularly GLCOM is a symmetry matrix; its level is determined by the image gray-level. Elements in the matrix are computed by the equation showed as follow:

$$
P(\mathrm{i}, j \mid d, \theta)=\underset{\Sigma \Sigma P(\mathrm{i}, j \mid d, \theta)}{P(\mathrm{i}, j \mid d, \theta)}
$$

GLCOM expresses the texture feature according the correlation of the couple pixels gray-level at different positions. It quantificationally describes the texture feature. In this work, 
texture feature include four properties energy, contrast, entropy, homogeneity.

$$
\begin{aligned}
& \text { Energy } \mathbf{E}=\Sigma \Sigma \mathbf{p}(\mathbf{x}, \mathbf{y})^{\wedge} \mathbf{2} \\
& \text { It is a gray-scale image surface measure of } \\
& \text { homogeneity changing, reflecting the distribution of image } \\
& \text { grayscale consistency of weight and texture. }
\end{aligned}
$$

$$
\text { Contrast } I=\Sigma \Sigma(x-y) \wedge 2 p(x, y)
$$

Dissimilarity is the main oblique near the moment of inertia, which measure the value of the matrix is distributed and images of local changes in number, reflecting the image clarity and texture of shadow depth. Contrast is large means texture is deeper.

Entropy $S=-\Sigma \Sigma p(x, y) \log p(x, y)$

The entropy measures image texture arbitrariness, when the space co-occurrence matrix for all values is equal, it achieved the minimum value; on the other hand, if the value of co-occurrence matrix is very irregular, its value is greater. Therefore, the maximum entropy implied by the image gray distribution is random.

Homogeneity

$$
\begin{aligned}
& H=\Sigma \Sigma 1 \mathrm{p}(\mathrm{x}, \mathrm{y}) \\
& 1+(\mathrm{x}-\mathrm{y})^{\wedge} 2
\end{aligned}
$$

It measures local changes in image texture number. Its value in large is illustrated that image texture between the different regions of the lack of change and partial very evenly. Here $p(x, y)$ is the gray-level value at the coordinate $(x, y)$.

The following Figure 1 shows how graycomatrix calculates several values in the GLCOM of the 4-by-5 image I. Element $(1,1)$ in the GLCOM contains the value 1 because there is only one instance in the image where two, horizontally adjacent pixels have the values 1 and 1 . Element $(1,2)$ in the GLCOM contains the value 2 because there are two instances in the image where two, horizontally neighboring pixels have the values 1 and 2. Graycomatrix continues this processing to fill in all the values in the GLCOM.

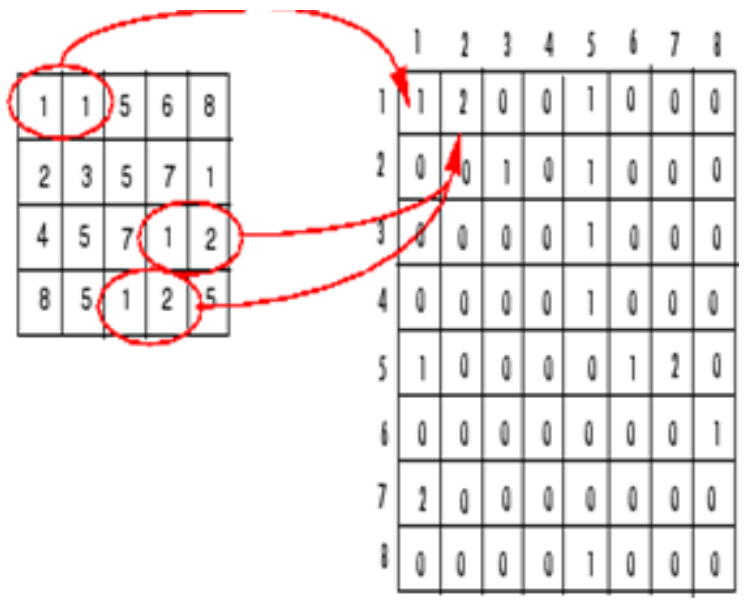

Figure 1: Graycomatrix for 4-by-5 image

\section{PROPOSED SCHEME}

\subsection{System Purpose}

Even though the measure of study in Sketch-Based Image Retrieval (SBIR) increases, there is no widely used SBIR system. The main aim is to develop a content-based associative search engine, which databases are available for anyone looking back to sketchy drawing. The important task is to bridge the information gap between the drawing and the picture, which is helped by own preprocessing transformation process. The block diagram of SBIR is shown below as shown in Figure 2.

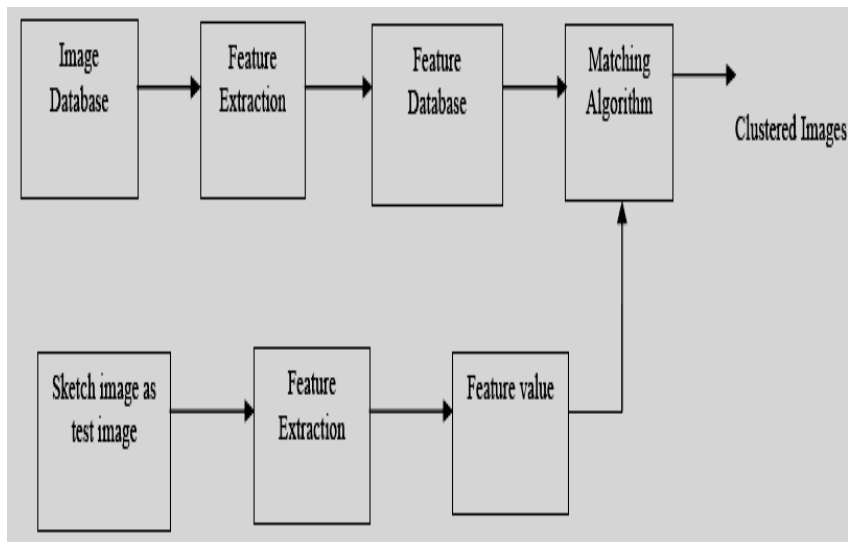

Figure 2: SBIR Block diagram

The content-based retrieval as a process can be divided into two main phases. The first is the database construction phase, in which the data of preprocessed images is stored in the form of feature vectors. This part carries out the computation rigorous tasks, which has to be done before the program actual use. The other phase is the Retrieval process, which is the on-line unit of the Program. 


\subsection{Preprocessing Subsystem}

In the preprocessing system feature vector is generated. For the image database feature values are generated by feature mining. Preprocessing subsystem is shown in Figure 3.

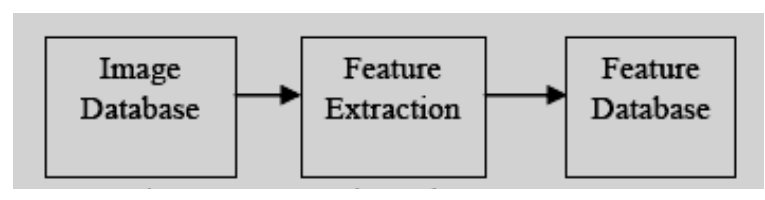

Figure 3: Preprocessing subsystem

Before the feature extraction, the images in the database need to be transformed into another form. The database consists of color images. In the retrieval process the color images must be transformed into gray type images. RGB images are converted into gray scale images. For the gray images features extracted. The GLCOM is used for feature extraction. For the image retrieval process the feature database is needed where it contains the extracted values. First, the image database is taken and project is executed. By using the GLCOM technique texture feature is extracted for each image of the image database. The four properties of GLCOM (Energy, Contrast, Entropy, and Homogeneity) are extracted for each image. The extracted texture feature values are stored in feature database which is needed further. This feature database is generated in preprocessing system which is the off-line part of the program. This feature database is additional to the program.

\subsection{Image Retrieval Process}

The main objective of our program is the sketch based image retrieval system, sketch image is used as query. In this work the query sketch was taken by translating original image into sketch image. All the necessary sketch images were taken by translating their original images into sketch form. For translation photo to sketch converter software was used. These sketch images are given as query in the program while execution process. The texture feature was used for sketch to generate feature value. The sketch image was taken as query image. The four GLCOM property values are calculated and stored as feature value for sketch image for each execution. The feature database which was created at preprocessing subsystem is added to the system and the four property values of the sketch image are compared with the values in the feature database. For the retrieval distance based search was used. The images and their feature values are stored and necessary mechanism for subsequent processing is provided. This is the database management subsystem, which consists of three parts, the storage, the retrieval, and the data manipulation modules [3].

The storage component provides images, data and the associated feature vectors are uploaded to the database. The file name, size and format of the image are emotionally involved. The sketch image is given as query to the program. The texture feature is extracted for the sketch image. The sketch image feature values are compared with the feature database. The www.ijsea.com comparison between texture values of the sketch image and database can be done by using distance metric. This assessment will be done at matching algorithm. The matching algorithm provides distance metric. The Euclidean Distance metric for similarity measurement [4] are used. It is given by the following equation:

The Euclidean distance between point's $\mathbf{p}$ and $\mathbf{q}$ is the length of the line segment connecting them ( ).

$$
D(p, q)=\operatorname{sqrt}(\Sigma(q i-p i) \wedge 2)
$$

The comparison between the sketch image and image database is measured by comparing distance values and relevant clustered images are displayed. The number of results to show in the user interface is an important aspect. Prima facie the first ' $n$ ' pieces of results can be displayed, which easily can be placed in the user interface. This number depends on the resolution of the monitor, and forasmuch the large resolution monitors are widely used, so this number can move between 20 and 40. Our program has been written in MATLAB, and during the implementation some new idea of was considered.

\section{EXPERIMENTATION AND RESULTS}

\subsection{Used Test Databases}

The system was experienced with more than one sample database to obtain a more extensive description of its positive and negative properties. The Wang database was used, which contains 100 images. The images can be divided into 10 classes based on their content, namely dinosaurs and mountains and another database is the Cambridge database which contains 60 images. In this work the combination of Wang database and Cambridge database is used for test purpose. The rotated images of original images for the test purpose since GLCOM is rotation invariant method which was used for feature extraction. All the images in the combined group are with the $816 \times 616$ resolution. These images are stored in JPEG format.

\subsection{Performance Measurement}

The efficiency of the system forming methods, and compare the different applied methods. The performance measurements are defined, evaluated and which method works effectively in what circumstances, and when not can be determined.

Let be a test database containing $\mathrm{N}$ pieces images, $\mathrm{P}$ length retrieval list, from which $\mathrm{Q}$ pieces matter as relevant results, and $\mathrm{Z}$ denotes the number of expected relevant hits.

Precision $=$ No. of images displayed with similar shape $(\mathrm{Q}) /$ No. of images displayed $(\mathrm{P})$

Where, the precision gives information about the relative effectiveness of the system. 
Recall $=$ No. of images displayed with similar shape $(\mathrm{Q}) /$ No. of images with similar shape in whole database (Z)

Where, the recall gives information about the complete accuracy of the system. The combination of Wang database and Cambridge database in two forms are taken: The first form is the combination of Cambridge database images and rotation versions of Wang database images. The second form is the combination of Wang database images and rotation versions of Cambridge database. The results have been shown for four different sketch images in Figures 4 and 5. They produce the results of the proposed method for first database

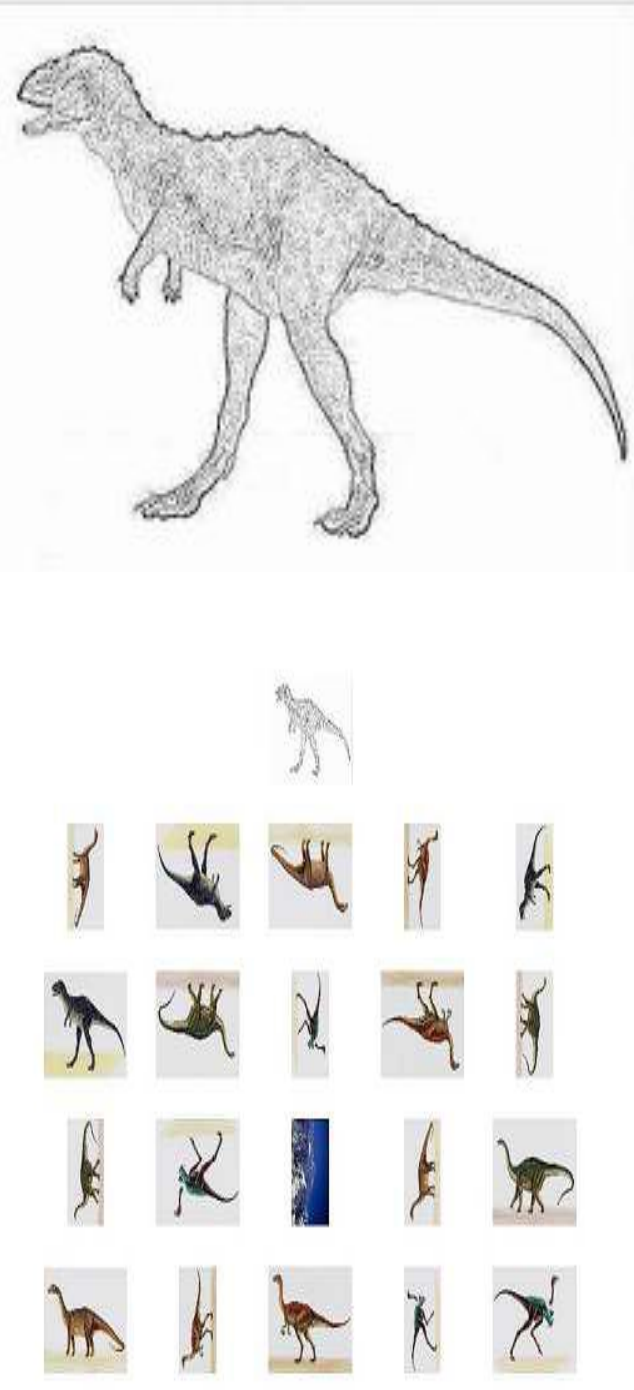

Figure 4: Shows Top 20 retrieved images based on Dinosaur sketch image as query image
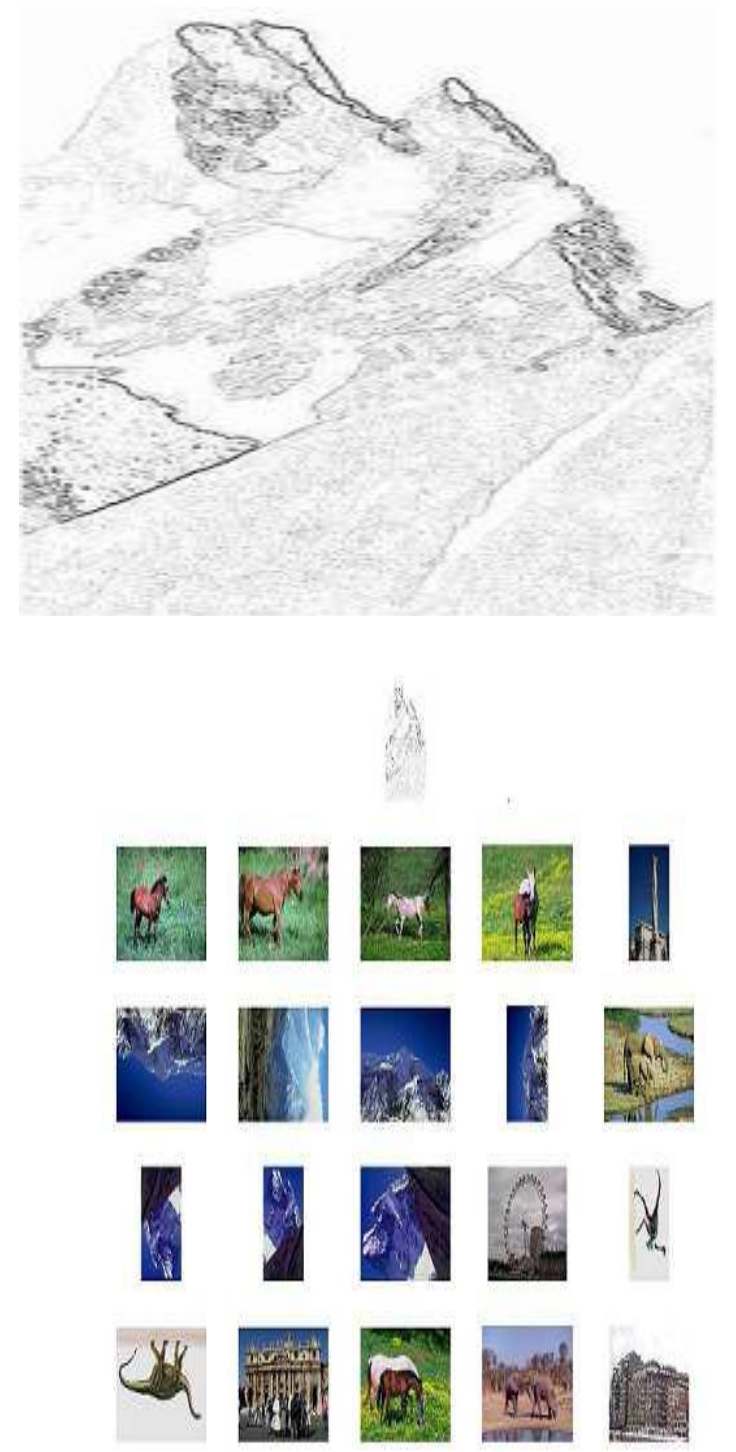

Figure 5: Shows Top 20 retrieved images based on Mountain sketch image as query image

The act of SBIR described in terms of precision and recall values. For the Dinosaur sketch image which is shown in Figure 4, the performance rate is high. In the whole database we have 20 rotated versions of dinosaur images. From those images 19 dinosaur images are retrieved. The retrieval rate is high by using the dinosaur image is texture dominant image. The GLCOM method for image retrieval is used to retrieve the texture dominant images efficiently. So, for the dinosaur sketch image more no. of dinosaur images is retrieved. For left over sketch images Dinosaur, Mountain, which is shown in Figure 4 and 5, the performance rate is low. Since the GLCOM method which retrieve texture based images are used, the retrieval rate for these three images is low. 


\section{CONCLUSIONS}

In this work, system architecture for CBIR based GLCOM with the texture characteristics of the image retrieval has been proposed. In spite of the conventional text based image retrieval, SBIR is developed which gives best results. The implementation is done in the coarse level which gives the normal results. For more accurate results the hierarchical methods such as wavelets, edge detection, etc are used. The sketch based system allows users a sensitive access to searching tools. In this work, it is observed that the proposed method achieved low retrieval performance over there three images for sketch based and color dominant images showed in Figures 4 and 5. The future work will focus on improved retrieval performance of sketch and color dominant images by exploring additional image features.

\section{ACKNOWLEDGMENTS}

The author would like to express their sincere gratitude to the Management of LIET, Hyderabad for their constant encouragement and co-operation.

\section{REFERENCES}

[1] B S Manjunath, W Y Ma, "Texture feature for browsing and retrieval of image data", IEEE Transaction on PAMI, Vol 18, No. 8, pp.837- 842, 1996.

[2] D. Comaniciu, and P. Meer, "Robust analysis of feature spaces: color image segmentation," IEEE Conference on Computer Vision and Pattern Recognition, pp. 750-755, June 1997.

[3] T. Deselaers, D. Keysers, and H. Ney, "Feature for image retrieval: an experimental comparison," Information Retrieval, vol. 11, pp.77-107, December 2007.

[4] R. Fabbri, L.D.F. Costa, J.C. Torelli, and O.M. Bruno, "2D Euclidean Distance Transform Algorithms: a comparative survey, ACM Computing Surveys, vol. 44, pp. 1-44, February 2008.

[5] A.K. Jain, J.E. Lee, and R. Jin, "Sketch to photo matching: a feature-based approach," Proc. SPIE, Biometric Technology for Human Identification VII, vol. 7667, pp. 766702-766702, 2010.

[6] A.K. Jain, J.E. Lee, R. Jin, and N. Gregg, "Content based image retrieval: an application to Tattoo images," IEEE International Conference on Image Processing, pp. 2745-2748, November 2009.

[7] D. Squire, W. Mueller, H. Mueller, and J. Raki, "ContentBased Query of Image Databases, Inspirations from Text Retrieval: Inverted Files, Frequency-Based Weights and

www.ijsea.com
Relevance Feedback,"Proc. Scandinavian Conf. Image Analysis, pp. 7-11, 1999.

[8] W. Niblack et al., "The QBIC Project: Querying Images by Content Using Color, Texture, and Shape," in Proc. SPIE, vol. 1908, San Jose, CA, pp. 173-187, Feb. 1993. 